\title{
Therapeutic Intervention for Restoration of Sensory and Motor Function after Central Nervous System Injury
}

\author{
Jung-Ho Lee \\ Dept. Physical Therapy, Kyungdong Univ., Bongpo-ri, Toseong-myeon, Goseong- \\ gun, Gangwon-do, Korea \\ ljhcivapt@naver.com
}

\begin{abstract}
The objective of this study was to investigate the effects of extracorporeal shock wave therapy, on the expression of neurotrophin-3, and C-Fos in the central nervous system injury of rats. In this study, 16 eight-week-old, Sprague-Dawley-origin male white rats weighing 250-300g were tested. For low-intensity extracorporeal shock wave therapy, magnetic-type extracorporeal shock wave therapy equipment (HAEMIL, Soltar, Korea) was used. Extracorporeal Shock Wave Therapy (ESWT) was applied to the gastrocnemius muscle of the injured side hind limb. Western blot analysis was analyzed for the evaluation of the NT-3, and $C$-Fos. Before the experiment, the spinal cords of the experimental and control groups were extracted and pre-evaluated. Spinal cords were extracted after all experiments to extract neurotrophic factors from the experimental and control groups. The change amount of NT-3 and C-Fos expression was a statistically significant difference in the ESWT group that received shock waves compared to the control group. In the comparison of treatment effects of the therapeutic methods, the experimental group showed a significantly larger change compared to the control group.
\end{abstract}

Keywords: Sensory, Motor, Function, Therapy, Restoration

\section{Introduction}

Intracerebral Hemorrhage (ICH) sometimes develops voluntarily from continued chronic high blood pressure or secondarily develops due to cerebrovascular disease. Once intracerebral hemorrhage develops, blood accumulates inside the brain and destroys the Central Nervous System (CNS).

Primary brain damage occurs as pressure around the hemorrhage drastically increases. Secondary damage follows due to the change of brain parenchyma, including blood-brain barrier destruction, inflammation, and edema [1].

Like other adult diseases, intracerebral hemorrhage currently exhibits a decreasing incidence age and an increasing incidence rate. Hence, interest in methods for the prevention of intracerebral hemorrhage and therapy after its incidence is increasing. Recently, diverse therapeutic intervention methods have been introduced that are aimed at the recovery of symptoms due to intracerebral hemorrhage, including medicinal treatment, operative treatment, and physical therapy [2].

Article History:

Received (November 8, 2019), Review Result (December 12, 2019), Accepted (January 19, 2020) 
However, the conditions of patients vary according to their neurologic injury, and the progress of the disease differs because it is influenced by environmental factors. Hence, finding efficient rehabilitation therapy methods for intracerebral hemorrhage patients is difficult [3].

The objective of this study was to investigate the effects of Extracorporeal Shock Wave Therapy (ESWT) on the expression of neurotrophin-3 (NT-3), and C-Fos in central nervous system injury of rats.

\section{Subjects and methods}

\subsection{Subjects}

In this study, 16(eight-week-old, weighing 250-300g) male white rats were used. During the experiment, the laboratory environment maintained the temperature and humidity conditions of $22 \pm 5^{\circ} \mathrm{C}$ and $49 \pm 5 \%$. Four rats were assigned to each cage to minimize stress in space.

The experimental group $(n=8)$ received the ESWT after the intracerebral hemorrhage injury, whereas the control group $(n=8)$ did not receive therapy after the intracerebral hemorrhage injury [Figure 1].

All surgical procedures and experimental protocols followed the Institution of Animal Care and Use Committee (IACUC).

\subsection{Research methods}

\subsubsection{Extracorporeal shock wave therapy}

In this study, a magnetic-type shock wave device (HAEMIL, Soltar, Korea) was used to apply extracorporeal shock wave therapy. Extracorporeal shock wave therapy was applied to the gastrocnemius muscles on the injured side. In one treatment, extracorporeal shockwave treatments were applied 1000 times (frequency of $1 \mathrm{~Hz}$, energy flux density of $0.1 \mathrm{~mJ} / \mathrm{mm}^{2}$ ).

\subsubsection{Western blot analysis}

Western blot analysis was used for the evaluation of the NT-3, and C-Fos. Before the experiment, the spinal cords of the experimental and control groups were extracted and preevaluated. Spinal cords were extracted after all experiments to extract neurotrophic factors from the experimental and control groups.

\subsubsection{Statistical analysis}

For the statistical analysis in this study, the mean \pm standard deviation was described through descriptive statistics, using SPSS for Windows (version 18.0).

An independent sample t-test was used to examine the intergroup differences. The statistical significance level of all data was $\mathrm{p}<0.05$.

\section{Results}

Statistical analysis showed significant differences in NT-3 and C-Fos expression levels between groups $(\mathrm{p}<0.05)$. 
The change amount of NT-3 and C-Fos expression was statistically higher in the experimental group treated with extracorporeal shock waves than in the control group without any treatment [Figure 2][Figure 3].

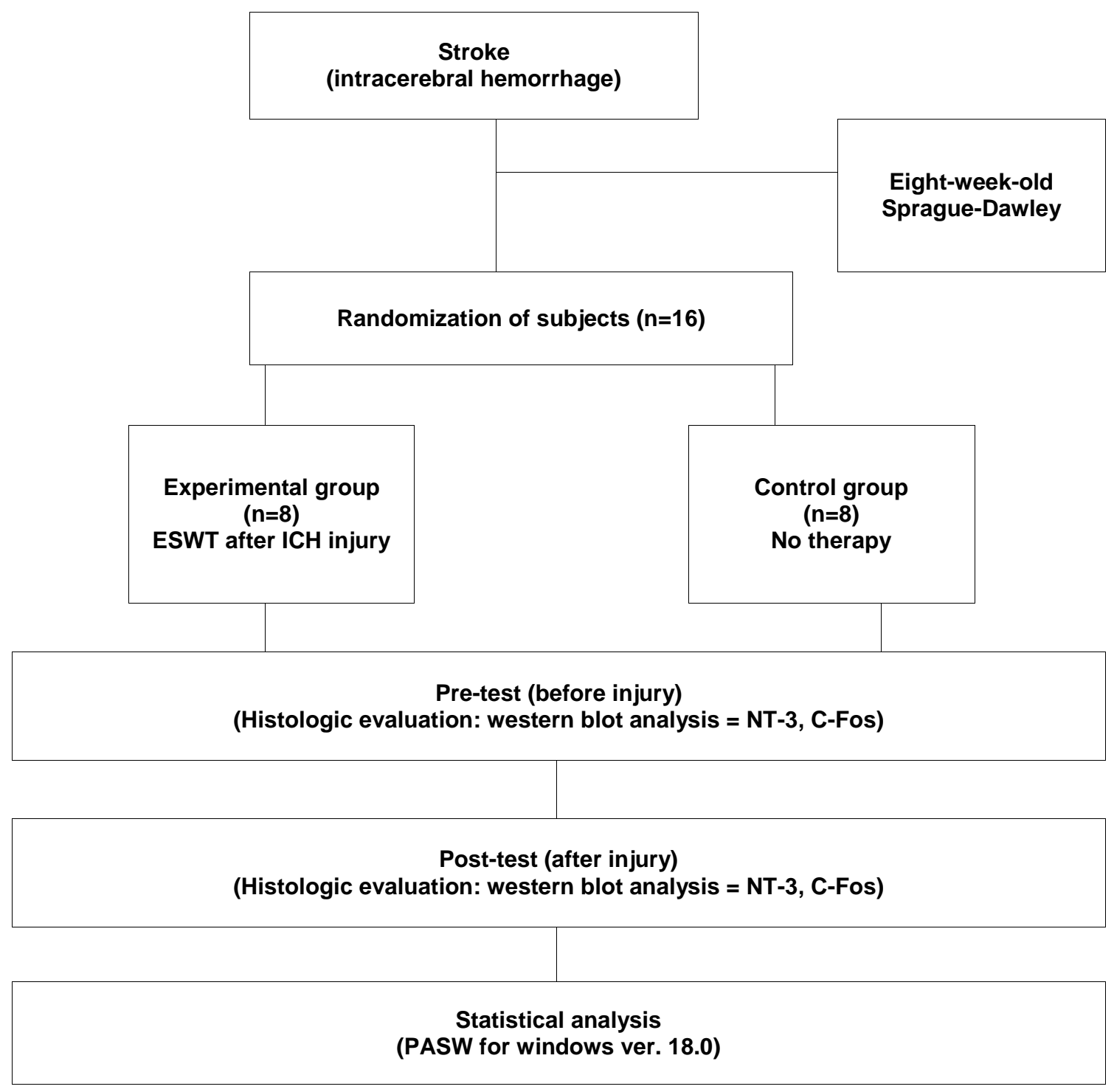

Figure 1. Flow chart

\section{Discussion}

Gait is a fundamental factor in human life, and patients with central nerve injury, such as damage to the descending motor pathway, have difficulties walking independently.

After a stroke, patients show asymmetrical sensorimotor cortex activity due to diminished activity of the affected side hemisphere during walking and excessive excitability of the corticospinal [4].

Because of these reasons, the gait of patients with stroke is limited in walking quality due to the occurrence of several defects, including the decrease of the affected side stride length, stance phase time, cadence, and gait speed, as well as an increase of spasticity [5]. 
Spasticity is a neurologic symptom that frequently develops after a stroke and it is defined as the muscle tone that increases according to speed and excessive tendon reflexes [6].

As spasticity causes pain and postural abnormalities and limits daily living activities and normal movement affecting the quality of life of patients, the appropriate therapy is required from the earliest stage of development [7].

Because central nerve injury is the main cause of spasticity, most studies regard excessively increased spinal excitability as a cause of spasticity [8].

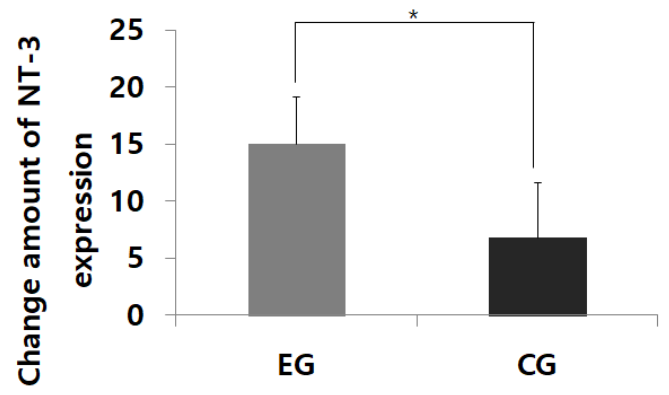

Figure 2. Comparison of NT-3 expression between groups * $\mathrm{p}<0.5$, Mean \pm SD: Mean \pm standard deviation, NT-3: Neurotrophin-3, EG: Experimental group, CG: Control group

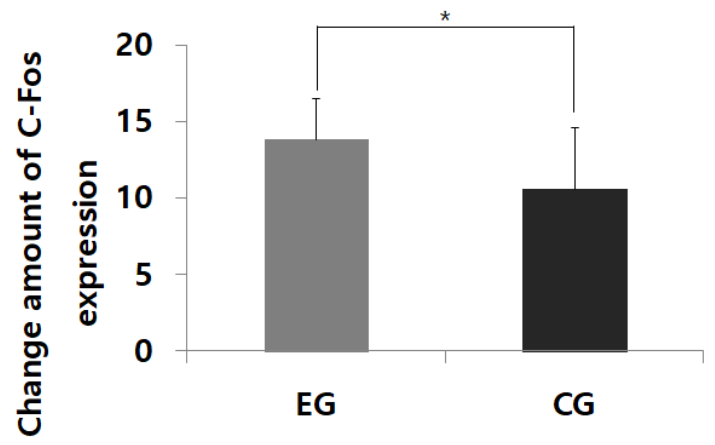

Figure 3. Comparison of C-Fos expression between groups * $<<0.5$, Mean \pm SD: Mean \pm standard deviation, EG: Experimental group, CG: Control group

This study examined the effects of applying ESWT to rats with central nerve injury on the expression of the NT-3 and C-Fos. In the study results, NT-3 expression was statistically significantly higher and C-Fos expression was statistically significantly lower in the experimental group compared to the control group. That is, ESWT promoted the expression of NT-3 and C-Fos which affected the regeneration, survival, and remodeling of nerves.

\section{Conclusion}

The chief goal of this study was to assess the effects of extracorporeal shock wave therapy for nerve regeneration, on the expression of neurotrophin-3 (NT-3), and C-Fos in the central nervous system injury of rats. 
To assess the effect of shock waves on the improvement of nerve regeneration, the extracorporeal shock wave was applied to the gastrocnemius muscle using an extracorporeal shock wave therapy device.

In the results of this study, the expression level of nerve growth factor affecting nerve regeneration was increased due to the application of extracorporeal shockwave therapy. That is, extracorporeal shockwave treatment applied after nerve injury has a positive effect on nerve regeneration.

In the next study, I hope that the use of extracorporeal shock wave therapy will demonstrate the effectiveness of treatment for a variety of diseases, symptoms, and sites.

\section{Acknowledgments}

This work was supported by the National Research Foundation of Korea (NRF) grant funded by the Korean government (MSIT) (No-2019R1F1A1057731).

\section{References}

[1] Y. Zhou, Y. Wang, J. Wang, R. Anne Stetler, and Q. W. Yang, "Inflammation in intracerebral hemorrhage: from mechanisms to clinical translation," Progress in Neurobiology, vol.115, pp.25-44, (2014) DOI: 10.1016/j.pneurobio.2013.11.003.

[2] H. R. Rostami, M. Akbarfahimi, A. M. Hassani, A. R. Akbarinia, and S. Samani, "Occupation-based intervention versus rote exercise in modified constraint-induced movement therapy for patients with median and ulnar nerve injuries: A randomized controlled trial," Clinical Rehabilitation, vol.31, pp.1087-1097, (2017) DOI: $10.1177 / 0269215516672276$.

[3] A. Santamato, A. Notarnicola, F. Panza, M. Ranieri, M. F. Micello, P. Manganotti, B. Moretti, F. Fortunato, S. Filoni, and P. Fiore, "SBOTE study: extracorporeal shock wave therapy versus electrical stimulation after botulinum toxin type an injection for a post-stroke spasticity-a prospective randomized trial," Ultrasound in Medicine \& Biology, vol.39, pp.283-291, (2013) DOI: 10.1016/j.ultrasmedbio.2012.09.019.

[4] F. Mori, G. Koch, C. Foti, G. Bernardi, and D. Centonze, "The use of repetitive transcranial magnetic stimulation (rTMS) for the treatment of spasticity," Progress in Brain Research, vol.175, pp.429-439, (2009) DOI: 10.1016/S0079-6123(09)17528-3.

[5] T. G. Kim, S. H. Bae, G. Y. Kim, and K. Y. Kim, "The effects of extracorporeal shock wave therapy on stroke patients with plantar fasciitis," Journal of Physical Therapy Science, vol.27, pp.523-526, (2015) DOI: 10.1589/jpts.27.523.

[6] S. Mense, and U. Hoheisel, "Shock wave treatment improves nerve regeneration in the rat," Muscle Nerve, vol.47, pp.702-710, (2013) DOI: 10.1002/mus.23631.

[7] T. Hausner, K. Pajer, G. Halat, R. Hopf, R. Schmidhammer, H. Redl, and A. Nógrádi, "Improved rate of peripheral nerve regeneration induced by extracorporeal shock wave treatment in the rat," Experimental Neurology, vol.236, pp.363-370, (2012) DOI: 10.1016/j.expneurol.2012.04.019.

[8] R. Mittermayr, V. Antonic, J. Hartinger, H. Kaufmann, H. Redl, L. Téot, A. Stojadinovic, and W. Schaden, "Extracorporeal shock wave therapy (ESWT) for wound healing: technology, mechanisms, and clinical efficacy," Wound Repair and Regeneration, vol.20, pp.456-465, (2012) DOI: 10.1111/j.1524475X.2012.00796.x. 
This page is empty by intention. 